\title{
A database of body-only computer-generated pictures of women for body-image studies: Development and preliminary validation
}

\author{
Joanna M. Moussally ${ }^{1,2}$ (D) Lucien Rochat $^{1,2} \cdot$ Andrés Posada $^{3} \cdot$ Martial Van der Linden ${ }^{1,2,4}$
}

Published online: 4 February 2016

(C) Psychonomic Society, Inc. 2016

\begin{abstract}
The body-shape-related stimuli used in most bodyimage studies have several limitations (e.g., a lack of pilot validation procedures and the use of non-body-shape-related control/neutral stimuli). We therefore developed a database of 61 computer-generated body-only pictures of women, wherein bodies were methodically manipulated in terms of fatness versus thinness. Eighty-two young women assessed the pictures' attractiveness, beauty, harmony (valence ratings), and body shape (assessed on a thinness/fatness axis), providing normative data for valence and body shape ratings. First, stimuli manipulated for fatness versus thinness conveyed comparable emotional intensities regarding the valence and body shape ratings. Second, different subcategories of stimuli were obtained on the basis of variations in body shape and valence judgments. Fat and thin bodies were distributed into several subcategories depending on their valence ratings, and a subcategory containing stimuli that were neutral in terms of valence and body shape was identified. Interestingly, at a descriptive level, the thinness/fatness manipulations of the bodies were in a curvilinear relationship with the
\end{abstract}

Electronic supplementary material The online version of this article (doi:10.3758/s13428-016-0703-7) contains supplementary material, which is available to authorized users.

Joanna M. Moussally

Joanna.Moussally@unige.ch

1 Cognitive Psychopathology and Neuropsychology Unit, Psychology Department, University of Geneva, Geneva, Switzerland

2 Swiss Center for Affective Sciences, University of Geneva, Geneva, Switzerland

3 Psychology Department, University of Geneva, Geneva, Switzerland

4 Cognitive Psychopathology Unit, Cognitive Sciences Department, University of Liège, Liège, Belgium valence ratings: Thin bodies were not only judged as positive, but also as negative when their estimated body mass indexes (BMIs) decreased too much. Finally, convergent validity was assessed by exploring the impacts of body-image-related variables (BMI, thin-ideal internalization, and body dissatisfaction) on participants' judgments of the bodies. Valence judgments, but not body shape judgments, were influenced by the participants' levels of thin-ideal internalization and body dissatisfaction. Participants' BMIs did not significantly influence their judgments. Given these findings, this database contains relevant material that can be used in various fields, primarily for studies of body-image disturbance or eating disorders.

Keywords Body-only pictures · Women ·

Computer-generated $\cdot$ Database $\cdot$ Development $\cdot$ Body image

\section{Introduction}

Body shape/weight plays an important role in societal beauty standards (Sentilles \& Callahan, 2012) and helps determine own-body image (Latner, 2012). Indeed, Western appearance norms promote thinness and deprecate fatness (Stice, 2002), a phenomenon associated with body dissatisfaction among women who dislike their bodies and struggle to achieve the thin ideal conveyed by the media (Stice \& Shaw, 2002). Bodyshape-related stimuli (e.g., referring to thinness vs. fatness) have therefore been used in a wide range of body-image studies focusing on the effects of exposure to societal appearance norms (for a meta-analysis, see Hausenblas et al., 2013), attentional biases (e.g., Gao et al., 2013; Glauert, Rhodes, Fink, \& Grammer, 2010), implicitly or explicitly assessed attitudes/ judgments toward body shapes/weights (e.g., Ahern, Bennett, \& Hetherington, 2008; Fan, Liu, Wu, \& Dai, 2004), body size estimation (e.g., Docteur, Urdapilleta, \& Rico Duarte, 2012), 
and memory biases (e.g., Gasperini \& Rousseau, 2014). However, the variety of stimuli used (e.g., words, pictures downloaded from the Internet, computer-generated pictures, schematic/drawn figures, or pictures of participants) has made the results of studies with similar objectives difficult to compare. In addition, the stimuli used in most studies in the field of body image had several limitations.

First, to the best of our knowledge, no validated database of body-shape-related stimuli is available, which means that researchers often create stimuli for their study objectives without pilot validation procedures. This might lead to the use of emotional stimuli (e.g., thin vs. fat) that are not comparable in terms of the emotional intensities they convey. For example, Glauert et al. (2010) found that the thin body they used in two studies was perceived as more extreme in terms of the body shape manipulation (i.e., a manipulation performed on a thinness/fatness axis) than the fat body, limiting some of their findings. To address these limitations, some researchers ensured that their thin and fat stimuli did not differ in terms of valence (e.g., Gao et al., 2013). Nevertheless, research about body shape often requires an examination of oppositely valenced stimuli (i.e., positive thin vs. negative fat stimuli), which requires ensuring that the stimuli are of comparable intensities (i.e., equally distant from the mean of the rating dimension).

Second, the control/neutral stimuli used have often no connection with the concept of body image/shape (e.g., the pictures of household objects selected by Gao et al., 2013). Thus, the possibility of differential processing (e.g., at the attentional, cognitive, or affective level) of relevant (bodies) and irrelevant (control) stimuli cannot be excluded. To overcome this limitation, the relevant (i.e., body-shape-related) control stimuli could be bodies rated as neither "thin" nor "fat" in terms of body shape and neither "positive" nor "negative" in terms of valence (i.e., bodies with a median/"zero-value" position between the two evaluation poles), meaning that they could be considered "nonemotional/neutral."

Third, the inherent properties of the stimuli used are also debatable. Indeed, depending on the research focus (e.g., attentional biases), words as stimuli have less ecological validity than visual stimuli (i.e., pictures). Schematic/drawn figures are also not very ecological, since they are less realistic than photographs or computer-generated pictures and convey the artist's subjective representation of body shapes (Gardner, Jappe, $\&$ Gardner, 2009). Likewise, pictures downloaded from the Internet often represent different people. Such stimuli must be matched for various features (e.g., gestures, clothing, and visual complexity), which can be complicated. Moreover, even when models can be matched for body mass index (BMI), they are still not the same person, meaning they have perceptibly different physical features (e.g., morphology). Consequently, pictures of the participants - which are nonetheless time-consuming to create-and computer-generated bodies present the advantage of overcoming these limitations, since they are visual and realistic, and allow the manipulation of a single body. Although the type of stimulus chosen is important, details are, too. For example, some researchers (e.g., Glauert et al., 2010) have used full bodies (from head to feet) or nude bodies. However, facial features can draw attention away from the body (Gardner et al., 2009), and nudity triggers specific attentional processing (similar to the processing of aversive stimuli; Most, Smith, Cooter, Levy, \& Zald, 2007).

\section{Overview of the present study}

To overcome some of the limitations discussed above, we developed a database of realistic computer-generated bodyonly pictures of women, wherein the body shapes were systematically manipulated in terms of fatness versus thinness. This database was specifically created to make validated stimuli available to researchers working in the field of body image. Given that computer-generated bodies are not affected by age, the database is principally designed for studies on relatively young participants. The pictures were therefore evaluated by a sample of young women (18-30 years old).

Normative data were obtained from rating scales directly related to body image - namely, attractiveness (judgments assessing the potential of a body to attract/repulse, underpinned by approach/avoidance tendencies), beauty (judgments assessing the potential of a body to be "pleasant/ unpleasant," underpinned by affective processes), harmony (judgments assessing the potential of a body to present a good balance or symmetry between its different parts, underpinned by evaluative processes), and body shape (judgments assessing a body on a thinness/fatness axis). Attractiveness, beauty, and harmony were subsequently reduced to a single dimension of valence (see the "Statistical analyses" section). To ensure that the manipulations performed on the stimuli were symmetrical, we tested whether our two general groups of stimuli (i.e., manipulated for fatness vs. thinness) were comparable in the emotional intensities they conveyed.

On the basis of normative data, we then explored the existence of stimulus categories (i.e., groups of stimuli triggering similar judgments), with the aim of giving researchers (a) different categories of pictures depending on judgment variations in both rating dimensions - namely, body shape and valence (e.g., positively assessed thin bodies); (b) information, if applicable, on different categories of stimuli that would be comparable on one rating dimension (e.g., negatively assessed fat bodies and negatively assessed thin bodies), meaning that these categories would convey comparable emotional intensities; (c) information on stimuli that would receive a median judgment on the two rating dimensions (i.e., neither "thin" nor "fat" and neither "positive" nor "negative"), meaning that 
they would be judged as neutral, and therefore could be used as control stimuli in future research.

Finally, the convergent validity of the pictures was assessed by exploring the impact of body image on participants' judgments, through well-known related variables such as thinideal internalization, body dissatisfaction, and BMI. These three body-image-related variables were selected because they all play roles in the development and maintenance of a negative body image (Cash, 2012) and have been shown to impact participants' judgments of bodies. For example, Glauert, Rhodes, Byrne, Fink, and Grammer (2009) demonstrated that participants with higher levels of body dissatisfaction or thinideal internalization had thinner body ideals (i.e., assessed thinner bodies positively) than did participants with lower levels. Similarly, participants with higher BMIs chose higher-BMI ideals (i.e., assessed larger bodies positively; Naghshizadian et al., 2014). We therefore explored whether these three body-image-related variables impacted participants' judgments of our computer-generated body-only pictures.

\section{Method}

\section{Development of stimuli}

Using the 3-D modeling software DAZ Studio 4.0 (DAZ Productions, 2011), 61 photorealistic women's bodies were created. The basic female model offered by the software ("Genesis," female value $=1.00$ ) was manipulated, with a sensitivity of 0.10 , on the dimension of thinness/fatness (the standard "thin" or "heavy" slider was increased in steps of 0.10 from 0.00 to 3.00 ) to obtain a continuum of bodies from extreme emaciation (30 bodies gradually manipulated for thinness) to morbid obesity ( 30 bodies gradually manipulated for fatness). The volumes of the 3-D female models were assessed using Voxelizer (Morris, 2013). The following formula was applied to estimate their BMIs: BMI $=[($ volume $*$ $0.85 / 1,000) /$ height $\left.^{2}\right]$, where 0.85 is a density correction coefficient based on calibration tests, ${ }^{1}$ and 1,000 is a divisor that converts the assessed volume to an estimated weight by adjusting the measurement units. The height of the 3-D

\footnotetext{
${ }^{1}$ A first phase of calibration tests of Voxelizer was performed with geometric figures (whose volumes were known). It revealed that the software slightly overestimated the actual volumes (by adding voxels to the circumference of the figure). A second phase consisted in a pilot study ( $N=$ 15 ; ten women, five men). The participants were instructed to estimate the BMIs of five female models (the basic model provided by DAZ Studio, two models manipulated for thinness, and two models manipulated for fatness) with the help of a standard figure-rating scale, with BMIs ranging from 17.5 to 40.00 . The difference between the participants' mean estimations and those obtained with Voxelizer indicated an average correction coefficient of 0.85 , close to the one obtained in the first phase of calibration tests with geometric figures.
}

models was defined as $1.65 \mathrm{~m}$ (i.e., close to the average height of European women; Cavelaars et al., 2000). Their estimated BMIs ranged from 13.19 (very severe underweight; estimated weight $=35.90 \mathrm{~kg}$ ) to 120.29 (very severe obesity; estimated weight $=327.50 \mathrm{~kg}$; World Health Organization [WHO], 1995, 2000, 2004). ${ }^{2}$ The models were dressed in white panties and undershirts. After export to a 2-D format, their heads were removed, using Adobe Photoshop CS5 (Adobe Systems, 2010), to focus attention on their body features (e.g., the thinness/fatness of the body shapes) and to avoid any impact of facial features (as was recommended by Gardner \& Brown, 2010; Gardner et al., 2009). Supplemental Material 1 illustrates representative examples of the stimuli. All 2-D stimuli and an explanatory file (StimuliInformation.pdf) can be downloaded from the Web (www. unige.ch/fapse/PSY/groups/upnc/BodyImageStimuli.zip).

\section{Stimulus validation}

\section{Participants}

The study included 82 young women. They were recruited from the community in the French area of Switzerland, with advertisements displayed around the University of Geneva campus and posted on social networks. All participants were volunteers and gave their written consent prior to their participation. The study protocol was approved by a local ethics committee and carried out according to the 1964 Declaration of Helsinki. All participants were native or fluent French speakers and had normal or corrected-to-normal vision. The mean age of the sample was 22.57 years $(S D=2.47$, range $=$ 18-30 years), and their mean BMI was $21.93(S D=3.05$, range $=17.51-30.48 \mathrm{~kg} / \mathrm{m}^{2}$ ).

\section{Measures}

Personal information sheet Participants answered several questions about their sociodemographic and anthropometric data (e.g., age, height, weight, mother tongue, and mastery of French if it was not their mother tongue). Participants' self-reported heights and weights were used to calculate their BMIs.

Stimulus rating scales Four 9-point Likert rating scales were created for this study to obtain evaluations of the 2-D stimuli directly linked to this study's area of interest (i.e., body

\footnotetext{
${ }^{2}$ The estimated weights and BMIs for the two extreme endpoint models could seem extreme, but they were designed so that the database could be used with different populations: samples from the community, but also clinical samples (e.g., individuals with anorexia nervosa or obese people). Therefore, the continuum of bodies contained in the database needed to be wide enough (i.e., to contain extreme and rare BMIs) in order to avoid ceiling or floor effects in clinical samples.
} 
image). The four rating scales allowed general aspects of a body to be described on several dimensions: (a) attractiveness $(1=$ repulsive to 9 = attractive $)$ (b) beauty $(1=u g l y$ to $9=$ beautiful); (c) harmony $(1=$ deformed to $9=$ harmonious $)$; and $(\mathrm{d})$ body shape $(1=$ fat to $9=$ thin $){ }^{3}$ Each rating scale was preceded by the following instructions: "Indicate how you rate this woman's body on the scale."

Stimulus evaluation task The 61 body-only 2-D pictures were evaluated by the participants in a computerized task. In this task, a stimulus appeared on the screen accompanied by one of the four Likert rating scales and remained on the screen until the participant had evaluated it on the rating dimension (i.e., attractiveness, beauty, harmony, or body shape). Each picture was evaluated on all four rating dimensions before the next picture appeared on the screen. For each stimulus, the four rating scales were ordered randomly. This procedure was repeated until all 61 randomly ordered stimuli had been evaluated.

Body-image questionnaires Thin-ideal internalization was assessed in terms of the total score of the Sociocultural Attitudes Towards Appearance Scale-3 (SATAQ-3; Thompson, van den Berg, Roehrig, Guarda, \& Heinberg, 2004; French validation: Rousseau, Valls, \& Chabrol, 2010), which measures attitudes toward societal standards of beauty and appearance promoted by the media. It is composed of 30 items scored on a Likert scale ranging from 1 (completely disagree) to 5 (completely agree). Its internal reliability in the present sample was excellent (Cronbach's $\alpha=.96$ ). We used the total score because the objective was not to explore the different SATAQ-3 dimensions of societal influences about thinness standards (i.e., the Internalization-General subscale measures the general internalization of the thin-ideal body promoted by Western societies; the Pressure subscale, the pressure of media to achieve the thin-ideal body; the Information subscale, the degree to which an individual perceives media messages as being relevant sources to determine ${ }^{3}$ The four rating scales relied on a large inventory (created with dictionaries, synonym lists, and French-language learning materials) of French adjectives describing general aspects of body shape (i.e., a body as a whole). A pilot study with 40 participants ( 24 women, 16 men) aged 19 to 68 years old showed [...] "?>19 to 68 years old showed that the eight adjectives (i.e., "repulsive" [repoussant]-"attractive" [attirant]; "ugly" [laid]-"beautiful" [beau]; "deformed" [difforme]-"harmonious" [harmonieux]; and "fat" [gros]-"thin" [mince]) used to create the poles of the four scales were well-known (responses on a Likert scale from 0 to 9; minimum mean $=6.05, S D=2.32,95 \% \mathrm{CI}=[5.31,6.79]$; maximum mean $=8.60, S D=0.84,95 \% \mathrm{CI}=[8.33,8.87])$, were perceived as antonyms (multiple-choice answers in a proposed list of adjectives, minimum percentage of correct antonym selection $=62.50 \%$; maximum $=$ $97.50 \%$ ), and were perceived as belonging to different categories of body description ("thinness" category: fat, thin; "beauty/attractiveness" category: repulsive, attractive, ugly, beautiful; "general appearance" category: deformed, harmonious; minimum percentage of correct attributions = $65 \%$, maximum $=100 \%$ ). his or her ideal body; and the Internalization-Athlete subscale, the internalization of the athletic-ideal body). Moreover, the construct of thin-ideal internalization is conceptually related to several SATAQ-3 dimensions (e.g., the thin-ideal internalization could be reinforced by the pressure of the media and by the perception of media messages as being relevant to our own beauty standards). Finally, the total score and the Internalization-General subscale (which, by definition, should focus on the internalization of the thin-ideal body) were highly correlated $(r=.92, p<.001$, in the present sample), highlighting that they probably assessed the same construct. For body dissatisfaction, we used the Body Shape Questionnaire (BSQ; Cooper, Taylor, Cooper, \& Fairburn, 1987; French validation: Rousseau, Knotter, Barbe, Raich, \& Chabrol, 2005), which evaluates concerns and feelings toward one's own body shape/weight using 34 items. Answers are given on a 6point Likert scale ranging from 1 (never) to 6 (always). Its internal consistency in the present sample was likewise excellent (Cronbach's $\alpha=.97)$.

\section{Procedure}

Participants were tested individually in a quiet room. After informed consent was obtained, they first completed the personal information sheet. Next, they evaluated the 61 bodyonly 2-D pictures using the four Likert rating scales, and then completed the two body-image questionnaires. Finally, they were debriefed about the objectives of the study.

\section{Statistical analyses}

Given that the judgments of the stimuli obtained on the dimensions of attractiveness, beauty, and harmony were strongly correlated $(r$ ranges $=.94-.98$, all $p \mathrm{~s}<.001)$, they were grouped - by obtaining their mean - into a single dimension called valence (Cronbach's $\alpha=.99$ ). All statistical analyses were therefore performed with two judgment dimensions: valence and body shape. These dimensions were moderately related $(r=.43, p<.001)$.

Our statistical analyses were divided into three parts. The first part consisted of analyses examining the participants' judgments of the 61 body-only pictures on the valence and body shape dimensions (i.e., descriptive statistics and distributions of the stimuli, as well as differences between the bodies manipulated for fatness and the bodies manipulated for thinness). The second part focused on an examination of the different stimulus categories based on valence and body shape judgments (i.e., category definitions and differences in judgments between the categories). Finally, the last part explored the impact of body image, through related variables (i.e., body dissatisfaction, thin-ideal internalization, and participants' BMIs), on valence and body shape judgments. 


\section{Results}

\section{Stimulus evaluation}

Normative data (descriptive statistics) for the 61 stimuli are reported in Supplemental Material 2, and graphical distributions of the stimuli for the two rating dimensions are illustrated in Fig. 1. On the body shape dimension, participants' judgments of the stimuli were distributed on a continuum of judgments from the extreme pole of fatness to the extreme pole of thinness (see Fig. 1). The unmanipulated body (i.e., the basic model offered by DAZ Studio 4.0, estimated BMI $=19.79$ ) was evaluated as being thin $(M=7.63, S D=1.26,95 \% \mathrm{CI}=$ $[7.36,7.91]){ }^{4}$ Depending on the degree of manipulation, the stimuli manipulated for fatness $(N=30)$ were considered to range from thin (smallest manipulation: estimated $\mathrm{BMI}=$ $21.55, M=7.28, S D=1.25,95 \% \mathrm{CI}=[7.01,7.56])$ to extremely fat (largest manipulation: estimated BMI $=120.29, M$ $=1.05, S D=0.22,95 \% \mathrm{CI}=[1.00,1.10])$. The stimuli manipulated for thinness $(N=30)$ were considered to range from thin (smallest manipulation: estimated BMI $=19.61, M=$ $7.50, S D=1.39,95 \% \mathrm{CI}=[7.19,7.81])$ to extremely thin (largest manipulation: estimated BMI $=13.19, M=8.94, S D=$ $0.46,95 \% \mathrm{CI}=[8.83,9.04])$. Participants' judgments of the stimuli manipulated for fatness $(M=2.57, S D=0.56,95 \% \mathrm{CI}$ $=[2.45,2.69])$ differed significantly in their body shape assessments from judgments of the stimuli manipulated for thinness $(M=8.25, S D=0.62,95 \% \mathrm{CI}=[8.12,8.39])[t$ test for dependent samples $\left.{ }^{5}: t(81)=65.74, p<.001\right]$, revealing that general differences in the thinness/fatness of the body shapes were correctly perceived.

On the valence dimension, the stimuli manipulated for thinness and those manipulated for fatness were distributed on a continuum of judgments from positivity to negativity, depending on the degree of the manipulation performed (see Fig. 1). Specifically, the more the stimuli were manipulated for fatness or thinness (reaching very high or low estimated BMIs, respectively), the more negatively participants assessed them. Conversely, the less the stimuli were manipulated for fatness or thinness (presenting estimated BMIs in or around the normal range), the more positively participants assessed them (see Fig. 1 and Supplemental Material 2). Therefore, at a descriptive level, the manipulations performed (i.e., on the thinness/ fatness axis) on the stimuli presented a curvilinear relationship with the valence ratings (see Fig. 1). In line with societal norms (thinness promotion and fatness deprecation; Stice, 2002), participants' judgments of the stimuli manipulated for fatness ( $M$ $=3.07, S D=0.80,95 \% \mathrm{CI}=[2.90,3.25])$ were significantly more negative ${ }^{4}$ than those of the stimuli manipulated for

\footnotetext{
${ }^{4}$ The possible ranges of the mean values for valence and body shape dimensions were from 1.00 (negative judgment or fat body) to 9.00 (positive judgment or thin body).
}

thinness $(M=5.07, S D=0.89,95 \% \mathrm{CI}=[4.88,5.27])[t$ test for dependent samples $\left.{ }^{5}: t(81)=16.33, p<.001\right]$.

To test whether the two groups of stimuli (manipulated for fatness vs. thinness) were comparable in terms of the emotional intensities they conveyed, the variables of valence and body shape judgments were transformed into two new variables that operationalized the arousal conveyed by the stimuli. More specifically, from the participants' judgments, valence and body shape corresponded to bipolar variables (i.e., negativepositive, fat-thin). Therefore, to allow the comparison between the judgments for the two groups of stimuli (manipulated for fatness vs. thinness), participants' judgments were transmuted into unipolar scales (arousal for either valence or body shape), in which higher scores corresponded to higher emotional intensity. To obtain these variables, we calculated, for each stimulus, an arousal score by subtracting (a) the average stimulus judgment (the mean of all participants' judgments for a given stimulus) on a rating dimension (valence or body shape) from (b) the mean for this dimension. By obtaining the absolute value of this calculation, we were able to determine the distance of each stimulus from the means of the rating dimensions (valence or body shape), which corresponded to the emotional intensity conveyed by the stimulus for a rating dimension. We performed $t$ tests for independent samples $(N=60$ stimuli). The results revealed that, regarding the arousal they conveyed, the stimuli manipulated for fatness did not differ from the stimuli manipulated for thinness on either the valence dimension (mean diff. $=0.48,95 \% \mathrm{CI}=[-0.09,1.04]), t(58)=1.70$, $p=.095$, or the body shape dimension (mean diff. $=0.26,95 \%$ $\mathrm{CI}=[-0.28,0.80]), t(58)=0.96, p=.343$.

\section{Categories of stimuli}

In order to articulate the body shape judgments with the valence judgments, we explored the presence of different categories of stimuli. For each rating dimension, each stimulus was categorized as follows: (a) an average stimulus judgment (the mean of all participants' judgments for a given stimulus) that fell within a distance of \pm 1.5 around the dimension mean ${ }^{6}$

\footnotetext{
${ }^{5}$ A $t$ test for dependent samples was performed to take into account the within-group variation (i.e., interindividual differences between the participants), given that all participants performed all conditions of the independent variable of this analysis (repeated measures design; independent variable: stimuli manipulation [fatness vs. thinness]).

${ }^{6}$ For the valence dimension, $M=4.14, S D=2.53,95 \% \mathrm{CI}=[3.49$, 4.79]; for the body shape dimension, $M=5.46, S D=3.13,95 \% \mathrm{CI}=$ $[4.65,6.26]$. The choice of a distance of \pm 1.5 around the dimension mean was based on the idea that neutral (in this case, median) stimuli could be defined as stimuli for which judgments were at or near the midline or the mean of a rating dimension. The means of the rating dimensions were used as the "anchors" for the creation of categories, given that they were slightly distant from the midline of 5 . Therefore, it was more accurate to use the means than it was to use the midlines. Finally, the cutoff of \pm 1.5 was chosen because it allowed us to obtain a clear separation between stimulus categories (i.e., no overlap between the categories).
} 


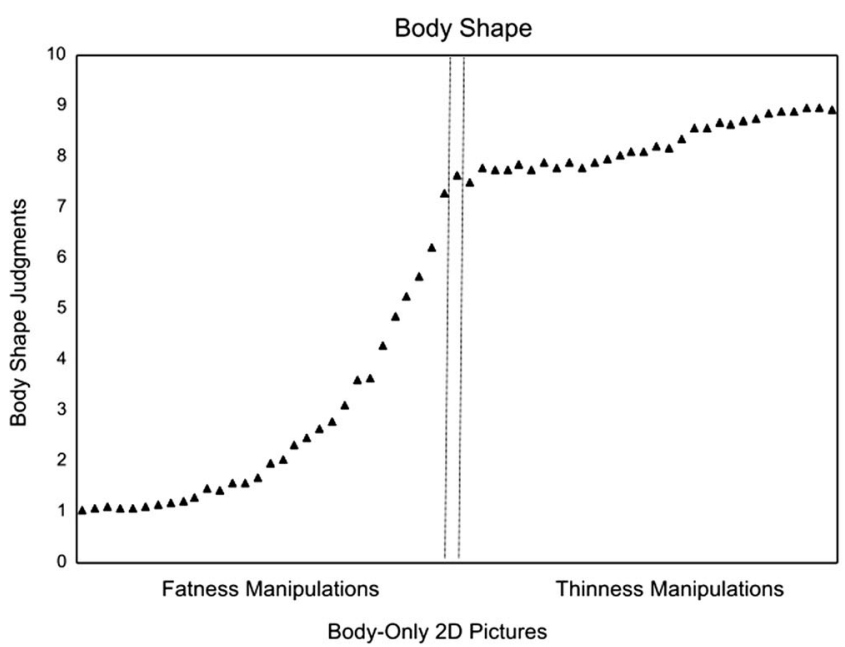

Fig. 1 Distributions of the participants' valence and body shape judgments of the 61 body-only 2-D stimuli. The stimulus within vertical lines, between the 30 bodies manipulated for fatness and the 30 bodies manipulated for thinness, corresponds to the basic model provided by the modeling software (i.e., the unmanipulated body). The stimuli closest to

was categorized as median; (b) an average stimulus judgment more than 1.5 from the dimension mean was categorized as

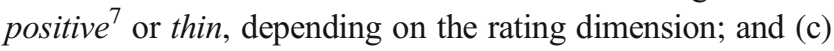
an average stimulus judgment more than -1.5 from the dimen-

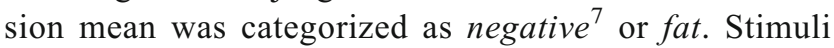
representing similar judgments were classified in the same category. Seven categories were obtained (see Fig. 2).

At the extreme of the fatness manipulation (estimated BMIs $\geq 53.21$, corresponding to very severe obesity; WHO, 2000), the bodies were judged as "fat" and "negative" $(N=$ 17). Less extreme bodies (estimated BMIs ranging from moderate [first obesity class] to very severe obesity; WHO, 2000) were judged as "fat" and "median" in terms of valence (i.e., neither positive nor negative; $N=7$ ). Then, bodies (estimated BMIs ranging from the overweight/pre-obese BMI class to the beginning of moderate obesity; WHO, 2000) received a "median" judgment on both rating dimensions (i.e., they were perceived as being neither thin nor fat and neither positive nor negative), meaning that they were evaluated as "neutral" $(N=$ 3 ). Once bodies thinned down (estimated BMIs around normal range; WHO, 2004), they were positively assessed up to a BMI of 16.12, corresponding to moderate underweight (WHO, 1995, 2004). Except for two bodies that received a median judgment in terms of body shape (neither thin nor fat; estimated BMIs around the upper bound of normal range; WHO, 2004), all were judged as thin $(N=17)$. Eventually, the thinner the bodies, the lower their valence judgments. Six bodies were judged as "thin" and "median" in terms of

\footnotetext{
${ }^{7}$ Given that the valence dimension included the evaluations of attractiveness, beauty, and harmony, a categorization as positive refers to bodies judged to be attractive, beautiful, and harmonious. A categorization as negative refers to bodies judged to be repulsive, ugly, and deformed.
}

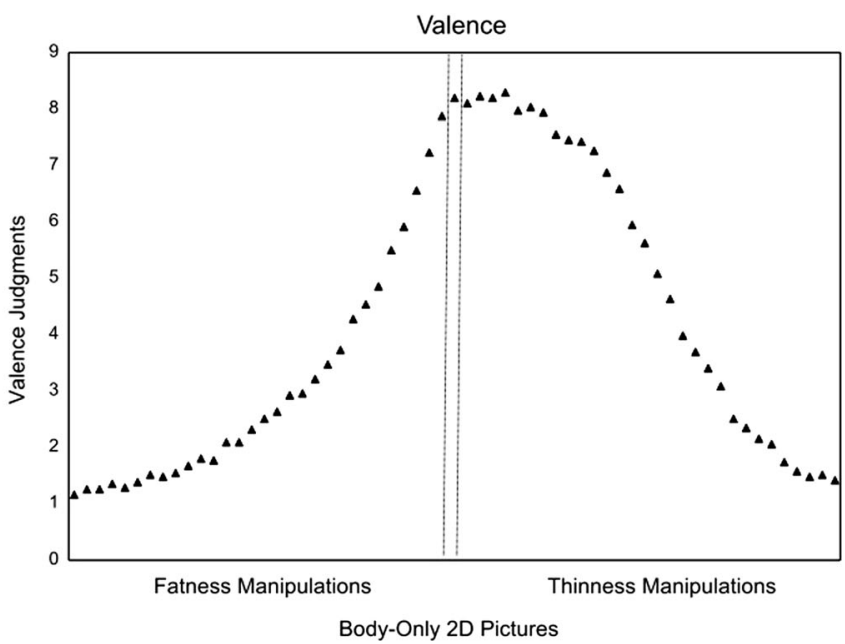

the unmanipulated body correspond to the smallest manipulations of fatness or thinness, and the stimuli to the extreme left or right of the distributions correspond to the largest manipulations of fatness or thinness. The possible scaling of valence and body shape judgments ranged from 1.00 (negative judgment or fat body) to 9.00 (positive judgment or thin body)

valence (estimated BMIs corresponding to severely underweight; WHO, 1995, 2004). The last category included bodies $(N=9)$ that were negatively assessed because of their extremely low BMIs (estimated BMIs $\leq 14.65$, corresponding to very severely underweight; Janghorbani \& Parvin, 1998), meaning that they were considered to be skinny/emaciated (i.e., "Thin-Negative" category of stimuli).

To ensure that participants' judgments for these categories differed (i.e., that there were separate categories), judgment differences between the categories were explored for the valence and body shape dimensions using Friedman's nonparametric analyses of variance (ANOVAs) with Wilcoxon posthoc tests. ${ }^{8}$ For the post-hoc tests, the conventional significance level was adjusted for multiple comparisons with the Bonferroni correction, resulting in a corrected alpha error level of $p=.002(p=.05 / 21)$. The results are illustrated in Fig. 3 . Overall, Friedman tests were significant for both the valence $\left[\chi^{2}(6)=425.43, p<.001\right]$ and body shape $\left[\chi^{2}(6)=463.56, p\right.$ $<.001]$ judgments. For the valence dimension, participants' judgments on the "Fat-Negative" and "Thin-Negative" categories did not differ significantly $(Z=0.48, p=.630)$, nor did judgments on the "Fat-Median" and "Thin-Median" categories $(Z=1.52, p=.129)$, meaning that these opposite categories on the body shape dimension had comparable valences. All other pairwise comparisons were significant (all $p \mathrm{~s}<$ .001). For body shape judgments, all pairwise comparisons were significant (all $p \mathrm{~s}<.001$ ), indicating that the stimulus categories were clearly perceived as distinct. Therefore, small differences in terms of the thinness or fatness of the body

\footnotetext{
${ }^{8}$ Nonparametric analyses were performed because the assumption of normality was violated for some judgment distributions in the ANOVA modalities/levels (i.e., stimulus categories).
} 


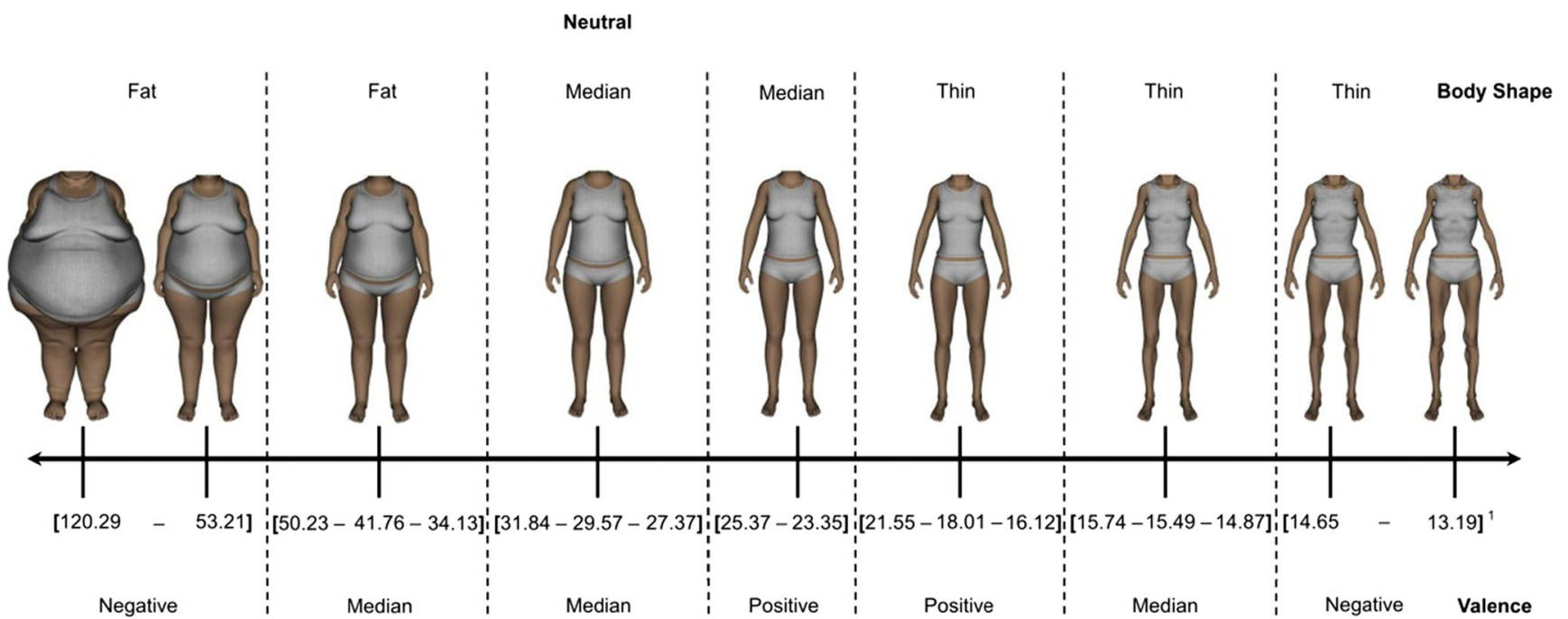

Fig. 2 Stimulus categories obtained on the basis of the observed variations in valence and body shape judgments: "Fat-Negative," $N=$ 17; "Fat-Median," $N=7$; "Neutral" (in terms of both body shape and valence), $N=3$; "Median-Positive," $N=2$, "Thin-Positive," $N=17$;

shapes of the stimuli (including, e.g., their estimated BMIs) were detected.

\section{Impact of body image on evaluation of stimuli}

Repeated measures analyses of covariance (ANCOVAs) were performed to examine the impacts of body-imagerelated variables (i.e., participants' levels of body dissatisfaction, thin-ideal internalization, and BMI) on valence and body shape judgments. The dependent variable was always one of the two rating dimensions: valence or body

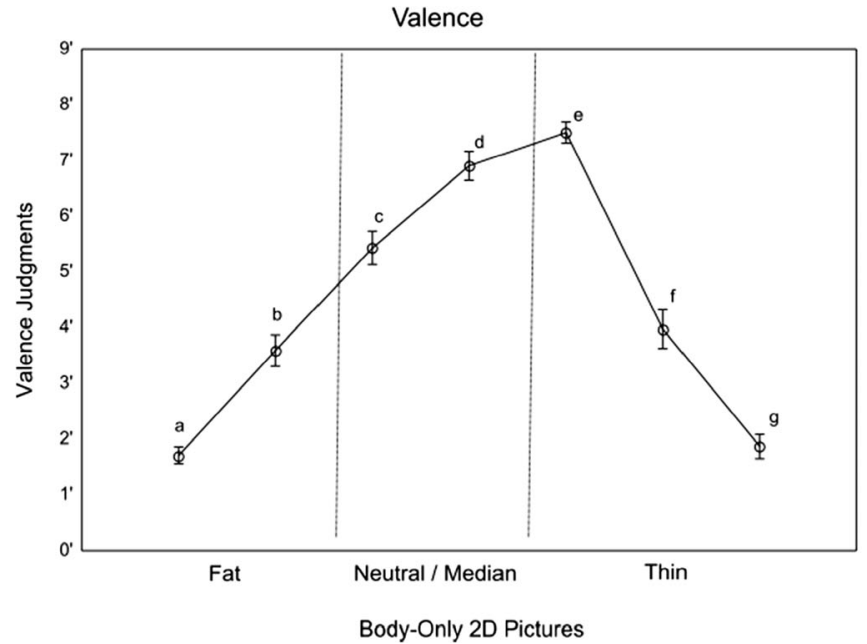

Fig. 3 Differences between stimulus categories, in terms of participants' judgments of valence and body shape $(N=82)$ : (a) "Fat-Negative," (b) "Fat-Median," (c) "Neutral" (in terms of both body shape and valence), (d) "Median-Positive," (e) "Thin-Positive," (f) "Thin-Median," (g) "Thin-Negative." For each category name, the first adjective corresponds to the body shape judgment, and the second, to the valence judgment. Vertical bars represent $95 \%$ confidence intervals. For the valence
"Thin-Median," $N=6$; "Thin-Negative," $N=9$. ${ }^{1}$ Ranges (with at least the upper and lower bounds) of the estimated body mass indexes of the stimuli in each category

shape. The within-subjects factor was the manipulation performed on the stimuli (thinness vs. fatness), and the independent variable (covariate) was body-image influence (BSQ, SATAQ-3, or BMI). The covariates were centered, and the interaction term (Within-subjects factor $\times$ Covariate) was computed with the centered covariates. The results are displayed in Table 1.

Concerning the valence judgments, in the three ANCOVAs (i.e., with the covariates BSQ, SATAQ-3, and BMI, respectively), the within-subjects factor was always significant (see Table 1), meaning that, regardless of their scores for the body-

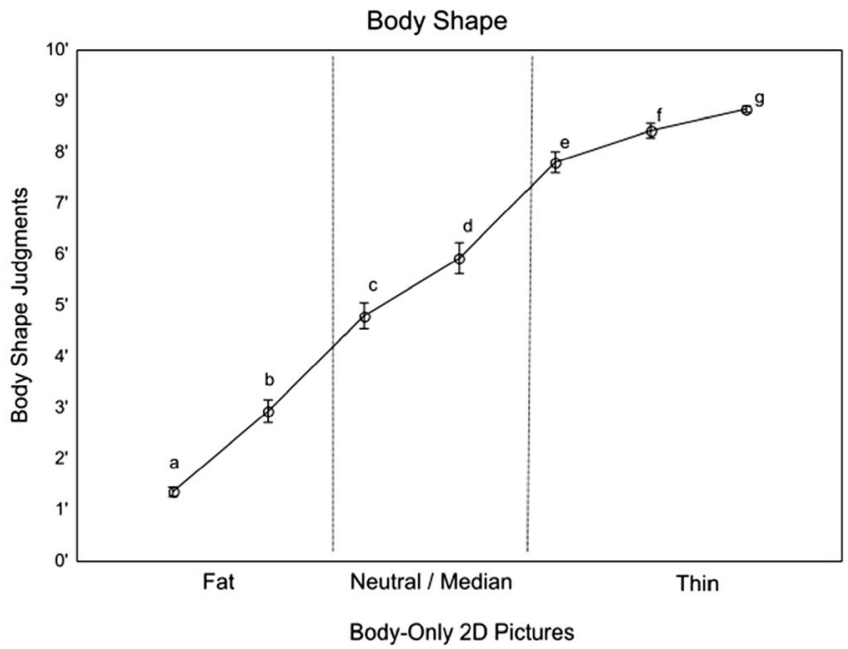

dimension, except for participants' judgments in the "Fat-Negative" (a) and "Thin-Negative" (g) categories and in the "Fat-Median" (b) and "Thin-Median" (f) categories, which do not differ, all other differences are statistically significant (all $p \mathrm{~s}<.001$; Wilcoxon post-hoc tests). For the body shape judgments, all differences are statistically significant (all $p$ s $<.001$; Wilcoxon post-hoc tests) 
Table 1 Repeated measures analyses of covariance for the impacts of body-image measures and participants' body mass indexes on valence and body shape judgments of the pictures

\begin{tabular}{|c|c|c|c|c|c|c|}
\hline & \multicolumn{3}{|c|}{ Valence Judgments } & \multicolumn{3}{|c|}{ Body Shape Judgments } \\
\hline & $F$ Value & $p$ Value & Partial $\eta^{2}$ & $F$ Value & $p$ Value & Partial $\eta^{2}$ \\
\hline BSQ & 1.13 & n.s. & .01 & 0.01 & n.s. & $<.01$ \\
\hline Type of evaluation ${ }^{1}$ & 297.83 & $<.001$ & .79 & $4,339.74$ & $<.001$ & .98 \\
\hline Type of evaluation ${ }^{1} \times$ BSQ & 10.45 & $<.01$ & .12 & 1.34 & n.s. & .02 \\
\hline SATAQ-3 & 1.34 & n.s. & .02 & 0.19 & n.s. & $<.01$ \\
\hline Type of evaluation ${ }^{1}$ & 308.15 & $<.001$ & .79 & $4,338.19$ & $<.001$ & .98 \\
\hline Type of evaluation ${ }^{1} \times$ SATAQ- 3 & 13.59 & $<.001$ & .15 & 1.31 & n.s. & .02 \\
\hline BMI & 0.08 & n.s. & $<.01$ & 1.27 & n.s. & .02 \\
\hline Type of evaluation ${ }^{1}$ & 269.91 & $<.001$ & .77 & $4,294.93$ & $<.001$ & .98 \\
\hline Type of evaluation ${ }^{1} \times$ BMI & 1.97 & n.s. & .02 & 0.50 & n.s. & $<.01$ \\
\hline
\end{tabular}

$N=82$. For all independent variables entered in the models, $d f=1$; for error, $d f=80 .{ }^{1}$ "Type of evaluation" corresponds to the valence or body shape judgments. BSQ = Body Shape Questionnaire; SATAQ-3 = Sociocultural Attitudes Towards Appearance Scale-3; BMI = body mass index

image-related variables, participants judged the stimuli manipulated for fatness significantly more negatively than the stimuli manipulated for thinness (see the "Stimulus evaluation" section above and Fig. 4). The main effect of the covariate was never significant. The two-way interaction was significant when the BSQ and SATAQ-3 were the covariates, but not when BMI was the covariate (see Table 1). Therefore, participants' BMIs did not significantly influence their valence judgments of the body-only pictures, whereas their body dissatisfaction or thin-ideal internalization did. When interpreting the significant two-way interaction with BSQ as the covariate, the parameter estimates revealed that the slope of the regression line was significant for stimuli manipulated for thinness $\left[b^{*}=.297, S E=.107 ; t(79)=\right.$ $2.79, p=.007]$, but not for stimuli manipulated for fatness $\left[b^{*}=-.142, S E=.111 ; t(79)=-1.28, p=.203\right]$. Thus, the

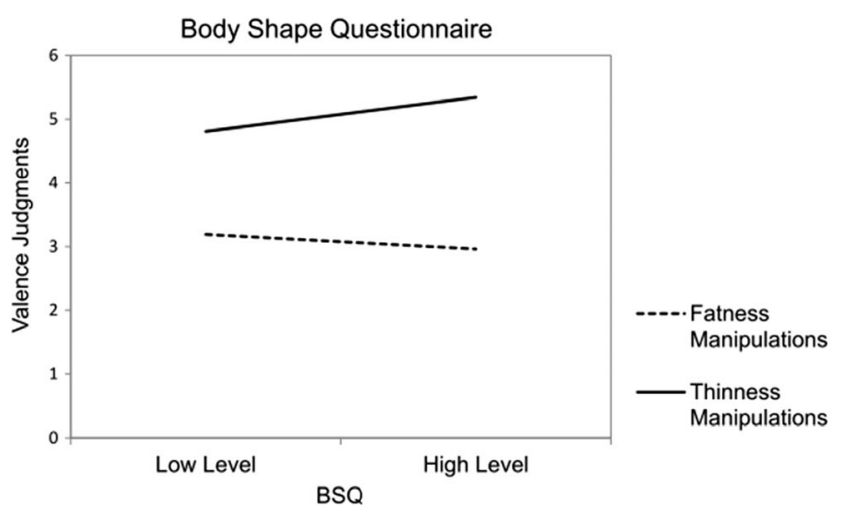

Fig. 4 Variations in valence judgments of stimuli manipulated for fatness versus thinness, as a function of participants' levels of body dissatisfaction (assessed with the Body Shape Questionnaire; BSQ) and thin-ideal internalization (assessed with the total score of the Sociocultural Attitudes Towards Appearance Scale-3; SATAQ-3). Regardless of participants' scores for the body-image-related variables, the valence higher was participants' body dissatisfaction, the more positively they judged the stimuli manipulated for thinness. This difference in valence judgments was not found for the stimuli manipulated for fatness. Regarding the significant two-way interaction with SATAQ-3 as the covariate, the parameter estimates again revealed that the slope of the regression line was significant for the stimuli manipulated for thinness $\left[b^{*}=.331\right.$, $S E=.106 ; t(79)=3.13, p=.002]$, but not for the stimuli manipulated for fatness $\left[b^{*}=-.162, S E=.110 ; t(79)=\right.$ $-1.47, p=.145]$. Thus, thin-ideal internalization replicated the results obtained with body dissatisfaction as a covariate: The higher was participants' thin-ideal internalization, the more positively they judged the stimuli manipulated for thinness. In summary, the valence judgments for stimuli manipulated for thinness - but not for those manipulated for fatness-became more positively polarized as a function of

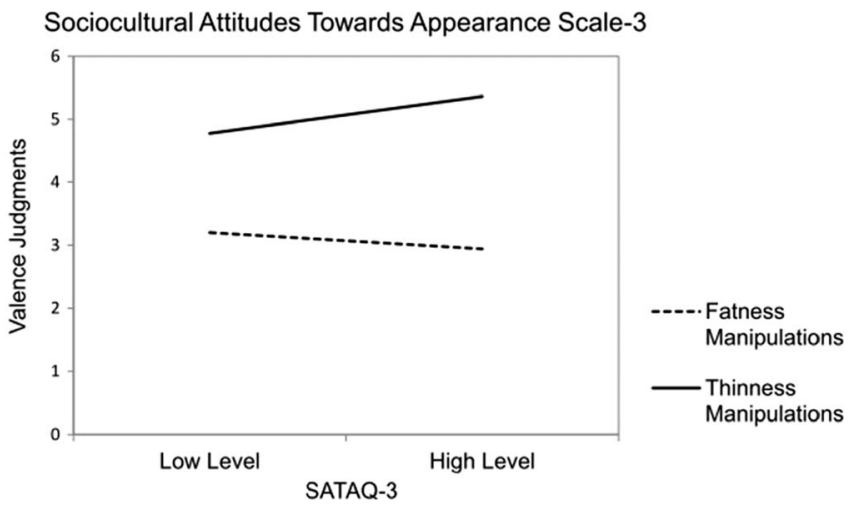

judgments of stimuli manipulated for fatness differed significantly from the judgments of stimuli manipulated for thinness (all $p \mathrm{~s}<.001$ ). Regression slopes for the fatness manipulations are not significant (all $p \mathrm{~s}>.05$ ); regression slopes for the thinness manipulations are significant (all $p \mathrm{~s}<$ $.01)$ 
participants' levels of body dissatisfaction or thin-ideal internalization. The two-way interactions are illustrated in Fig. 4.

Concerning the body shape judgments, in the three ANCOVAs (i.e., with the covariates BSQ, SATAQ-3, and BMI, respectively), the within-subjects factor was always significant, but the main effect of the covariate and the two-way interaction were never significant (see Table 1). Thus, participants' variations in body dissatisfaction, thin-ideal internalization, or BMI did not significantly influence their body shape judgments.

\section{Discussion}

The objective of this study was to develop and preliminarily validate a database of 61 computer-generated body-only pictures of women representing a continuum of body shapes (from extreme emaciation to morbid obesity). This database has many advantages. First, it contains realistic and ecological body-only pictures generated by the systematic manipulation of the same basic female body, which makes all of the bodies comparable (i.e., no differences in gestures, clothing, body morphology, or visual complexity). Second, it provides a large number of pictures with normative data, allowing researchers to select stimuli on the basis of their valence and/or body shape ratings, to suit particular research interests. Third, it contains neutral stimuli (in terms of valence and body shape) that can be used as control stimuli, and thus improve methodology (i.e., allowing the use of relevant emotional and nonemotional/neutral stimuli that are both related to the study's concept of interest).

The results revealed that the general thinness/fatness manipulations performed on the stimuli were correctly perceived: Stimuli manipulated for thinness were perceived as being thin, and stimuli manipulated for fatness were perceived as being fat. Moreover, the general judgments of stimulus valence were in line with Western beauty standards (e.g., Stice, 2002), in that the stimuli manipulated for fatness were assessed more negatively than the stimuli manipulated for thinness. These two general groups of 30 stimuli, manipulated for either thinness or fatness, were comparable in terms of the arousal they conveyed, meaning that the manipulations performed for thinness and fatness were equivalent.

Concerning the valence ratings of the various bodies, the body shape manipulations (i.e., performed on the thinness/ fatness axis) were not in a linear relationship with the valence dimension. In line with societal beauty standards, one might have expected such a linear relationship: that (a) the fatter the bodies were, the more negatively judged they would be and, reciprocally, (b) the thinner they were, the more positively judged they would be. But, on the contrary, our results showed a curvilinear relationship, with thin bodies being increasingly negatively judged after they exceeded a threshold BMI.
Variations in the judgments of body shape and valence generated different categories of stimuli that might be more suited for specific research interests than are the two broad categories. These separate categories revealed that, depending on the bodies' estimated BMIs, the stimuli manipulated for fatness were judged as negative or median in terms of valence, whereas the stimuli manipulated for thinness were judged as positive, median, and even negative, when their estimated BMIs became extremely low. The fat bodies categorized as negative or median were comparable in terms of valence to their thin counterparts. However, the thin bodies categorized as positive were not comparable to any other category. This is not surprising, given the valuing of thinness and the devaluing of fatness in Western societies (Stice, 2002). Nevertheless, these results raise questions about the control of stimulus valence judgments in past studies. More specifically, in order to have comparable fat and thin stimuli, past studies (e.g., Gao et al., 2013) probably used either (a) thin bodies that were either not very positively or almost negatively assessed, or (b) fat bodies that were very negatively assessed. In the first case, the stimuli would be thin and fat bodies with similar valences, contrary to the assumptions arising from Western beauty standards (e.g., Stice, 2002), which require one to examine oppositely valenced stimuli. In the second case, the thin and fat bodies would be oppositely valenced and trigger similar emotional intensities regarding valence, but they probably would not arouse similar emotional intensities regarding body shape, given that, at least in the present study, positively assessed thin bodies had estimated BMIs around the normal range, which the negatively assessed fat bodies did not. It would thus be difficult to compare positively assessed thin bodies (which represent ideal bodies) to a subgroup of fat bodies. Because it contains some neutral stimuli, our database will allow researchers to use control stimuli when positively valenced thinness is the focus of the study. Moreover, because of the large number of stimuli in the database, researchers can also create new stimulus categories more suited to their needs (e.g., by grouping positive and median thin bodies to constitute a group of moderately positive thin bodies that would be comparable in terms of valence to negative fat bodies).

Two interesting results regarding the stimulus categories should be discussed. First, thin bodies were positively assessed up to a moderately underweight BMI, illustrating the impact of the internalization of Western societies' thinness standards on participants' judgments of body shapes, as it has been pointed out by earlier studies focusing on the effects of exposure to thin or ideal bodies (e.g., Glauert et al., 2009; Stephen \& Perera, 2014). Second, the neutral bodies had BMIs that are considered to be overweight to moderately obese (BMI classes; WHO, 2000). This result may be explained by the prevalence of these BMIs among the Swiss population (overweight, $26 \%-28 \%$; obese, $4 \%-$ $16 \%$; Schutz \& Woringer, 2002); because these BMIs are 
common, they are probably less emotionally valenced than more obese bodies.

Finally, the impact of body-image-related variables revealed that higher levels of body dissatisfaction or thin-ideal internalization led to more positive judgments of stimuli manipulated for thinness, but did not significantly influence the body shape judgments of these bodies or any judgments of the stimuli manipulated for fatness. Thus, the perception of the thinness/fatness of body shapes was not affected, but the emotional intensity attributed to thin bodies was (becoming more extreme). These results are in line with the findings of Glauert et al. (2009), which underscored the impacts of body dissatisfaction and thin-ideal internalization on body-ideal ratings (i.e., thinner ideals among participants with higher scores for body-image-related variables). Moreover, these authors also observed a reduced impact of exposure to fat bodies for participants with higher scores for body-image-related variables. Concerning the impact of BMI on judgments, our results revealed that participants' BMIs did not significantly influence their valence or body shape judgments.

\section{Limitations}

Some limitations on this study should be mentioned. First, we cannot draw a conclusion about the nonsignificant results we obtained regarding the impact of BMI on the stimulus judgments. Indeed, these results do not necessarily mean that such effects were not present. In our sample, participants' BMIs ranged from mildly underweight to the beginning of moderate obesity. It is therefore possible that a person's BMI might impact his or her judgments of body stimuli only if the person has a lower or higher BMI than the range in our sample (e.g., moderately underweight or obese). For instance, in a sample with a large proportion of obese participants, Naghshizadian et al. (2014) showed that participants' BMIs did impact their body-ideal ratings (i.e., higher BMI ideals among participants with higher BMIs). Moreover, in this study, participants' BMIs were based on self-reported measures. We therefore cannot exclude the possibility that estimation biases were present and influenced the reliability of the measures, explaining the nonsignificant results. However, previous studies have highlighted that estimation biases are more likely to appear among people with "extreme" BMIs (e.g., Connor Gorber, Tremblay, Moher, $\&$ Gorber, 2007; Tovée, Emery, \& Cohen-Tovée, 2000). Since such BMIs were marginal in the present sample, we presuppose that estimation biases were rare.

Second, the database must be validated (e.g., through the development of normative data) in clinical samples (e.g., individuals with anorexia nervosa or obese people). Valence and body shape judgments could vary depending on sample characteristics, given that our results revealed that body image affects judgments of the stimuli. For instance, biases in size estimation - widely reported in samples of anorexic patients, who overestimate their body size (for a review, see Gardner \& Brown, 2014) - might impact body shape judgments, and ultimately valence judgments. We therefore encourage researchers to add a short stimulus evaluation task to their methodologies in order to obtain ratings of the stimuli in their particular sample, especially if they select a small number of bodies from our database. Indeed, the normative data presented in this study were obtained through an evaluation procedure in which participants had to judge bodies ranging from extreme emaciation to morbid obesity; their ratings were probably modulated in relation to the extreme bodies on the continuum (i.e., the anchors). More specifically, a stimulus located in the middle of the continuum was evaluated as being moderate by our sample because the participants probably compared it to the more extreme stimuli. However, if the continuum of bodies were changed (e.g., because of selection for a specific research interest), participants' ratings of the stimuli might be slightly modified, given that they would be based on different anchors.

Third, given that participants' ratings of the bodies could vary depending on the sample characteristics or stimulus selection, the utility of the stimulus categories could be questionable, because these categories were based on participants' ratings and could therefore also vary slightly (i.e., if the ratings of some bodies changed, this could impact the bodies that were grouped under the same category). Moreover, we did not compare the stimulus categories in terms of the emotional intensities (arousal) that they conveyed, which might have allowed us to observe whether the category of thin - positive bodies would be comparable to a category of fat bodies. Rather, the objective of the present study concerning the different stimulus categories was to describe them according to the rating dimensions, in order to illustrate the potential of our database: a continuum of bodies presenting a wide range of valence judgments in which the body shape (manipulations of body on a thinness/fatness axis) and valence dimensions are in a curvilinear relationship.

Fourth, as we mentioned above, these stimuli were principally designed to be used with relatively young Caucasian women participants. For studies with participants who are older or belong to another anthropological type, the methodology or stimuli will need to be adapted. For instance, to attenuate the impact of perceived ethnicity, the stimuli might be presented in grayscale (Swami, Cavelti, Taylor, \& Tovée, 2015). Furthermore, to control for the perceived differences between computer-generated bodies and participants' bodies, researchers could ask participants, using Likert scales, how different they find the shapes of the computer-generated bodies from their own.

\section{Future prospects}

The database of computer-generated body-only pictures of women has the advantage that it can easily be extended to 
better meet researchers' needs. For example, additional neutral bodies could be generated (by creating new bodies, using a smaller sensitivity of manipulation, with estimated BMIs within the range of bodies evaluated as neutral) in order to have comparable numbers of emotional and nonemotional/ neutral bodies. Similarly, more emaciated or obese bodies might be generated so that, in studies with clinical samples, anorexic or obese patients could have a larger continuum of computer-generated bodies similar to theirs.

Finally, our database was originally created to be employed in a wide range of research in the fields of body-image disturbance and eating disorders (e.g., studies focusing on attentional biases, effects of exposure to societal beauty norms, and body-size estimation). Nevertheless, it can be used more widely. Indeed, one might imagine using our stimuli in any study in which the body is the focus. In conclusion, this database has been made publicly available to overcome some limitations on the stimuli used in past studies of body image, but it may also interest researchers from various other fields.

Author note Many thanks to Joana Albuquerque Lopes and Lucile Combremont for their help with the data collection. The authors have no conflicting interests to disclose.

\section{References}

Adobe Systems, Inc. (2010). Adobe Photoshop CS5 Extended (Version 12.0.1) [Computer software]. San Jose: Adobe Systems, Inc.

Ahern, A. L., Bennett, K. M., \& Hetherington, M. M. (2008). Internalization of the ultra-thin ideal: Positive implicit associations with underweight fashion models are associated with drive for thinness in young women. Eating Disorders, 16, 294-307. doi:10.1080/ 10640260802115852

Cash, T. F. (2012). Cognitive-behavioral perspectives on body image. In T. F. Cash (Ed.), Encyclopedia of body image and human appearance (Vol. 1, pp. 334-342). London: Academic Press.

Cavelaars, A. E. J. M., Kunst, A. E., Geurts, J. J. M., Crialesi, R., Grötvedt, L., Helmert, U., ... Mackenbach, J. P. (2000). Persistent variations in average height between countries and between socioeconomic groups: An overview of 10 European countries. Annals of Human Biology, 27, 407-421. doi:10.1080/03014460050044883

Connor Gorber, S., Tremblay, M., Moher, D., \& Gorber, B. (2007). A comparison of direct vs. self-report measures for assessing height, weight and body mass index: A systematic review. Obesity Reviews, 8, 307-326. doi:10.1111/j.1467-789X.2007.00347.x

Cooper, P. J., Taylor, M. J., Cooper, Z., \& Fairburn, C. G. (1987). The development and validation of the Body Shape Questionnaire. International Journal of Eating Disorders, 6, 485-494. doi:10. 1002/1098-108X(198707)6:4<485::AID-EAT2260060405>3.0. $\mathrm{CO} ; 2-\mathrm{O}$

DAZ Productions. (2011). DAZ Studio (Version 4.0.3.47) [Computer software]. Draper: DAZ Productions.

Docteur, A., Urdapilleta, I., \& Rico Duarte, L. (2012). The role of cognitive factors in body-size perception and recall-size estimation in normal-weight women. Revue Européenne de Psychologie Appliquée, 62, 129-135. doi:10.1016/j.erap.2012.05.001
Fan, J., Liu, F., Wu, J., \& Dai, W. (2004). Visual perception of female physical attractiveness. Proceedings of the Royal Society B, 271, 347-352. doi:10.1098/rspb.2003.2613

Gao, X., Li, X., Yang, X., Wang, Y., Jackson, T., \& Chen, H. (2013). I can't stop looking at them: Interactive effects of body mass index and weight dissatisfaction on attention towards body shape photographs. Body Image, 10, 191-199. doi:10.1016/j.bodyim.2012.12. 005

Gardner, R. M., \& Brown, D. L. (2010). Body image assessment: A review of figural drawing scales. Personality and Individual Differences, 48, 107-111. doi:10.1016/j.paid.2009.08.017

Gardner, R. M., \& Brown, D. L. (2014). Body size estimation in anorexia nervosa: A brief review of findings from 2003 through 2013. Psychiatry Research, 219, 407-410. doi:10.1016/j.psychres.2014. 06.029

Gardner, R. M., Jappe, L. M., \& Gardner, L. (2009). Development and validation of a new figural drawing scale for body-image assessment: The BIAS-BD. Journal of Clinical Psychology, 65, 113122. doi:10.1002/jclp.20526

Gasperini, C., \& Rousseau, A. (2014). Évaluation d'un biais de mémoire explicite chez les jeunes femmes françaises à risque de troubles des conduites alimentaires. Journal de Thérapie Comportementale et Cognitive, 24, 106-113. doi:10.1016/j.jtcc.2014.06.001

Glauert, R., Rhodes, G., Byrne, S., Fink, B., \& Grammer, K. (2009). Body dissatisfaction and the effects of perceptual exposure on body norms and ideals. International Journal of Eating Disorders, 42, 443-452. doi:10.1002/eat.20640

Glauert, R., Rhodes, G., Fink, B., \& Grammer, K. (2010). Body dissatisfaction and attentional bias to thin bodies. International Journal of Eating Disorders, 43, 42-49. doi:10.1002/eat.20663

Hausenblas, H. A., Campbell, A., Menzel, J. E., Doughty, J., Levine, M., \& Thompson, J. K. (2013). Media effects of experimental presentation of the ideal physique on eating disorder symptoms: A metaanalysis of laboratory studies. Clinical Psychology Review, 33, 168181. doi:10.1016/j.cpr.2012.10.011

Janghorbani, M., \& Parvin, F. (1998). Prevalence of overweight and thinness in high-school girls in Kerman, Iran. International Journal of Obesity, 22, 629-633. doi:10.1038/sj.ijo.0800638

Latner, J. D. (2012). Body weight and body image in adults. In T. F. Cash (Ed.), Encyclopedia of body image and human appearance (Vol. 1, pp. 264-269). London: Academic Press.

Morris, D. (2013). Voxelizer [Computer software]. Stanford: Stanford University Haptics Lab.

Most, S. B., Smith, S. D., Cooter, A. B., Levy, B. N., \& Zald, D. H. (2007). The naked truth: Positive, arousing distractors impair rapid target perception. Cognition and Emotion, 5, 964-981. doi:10.1080/ 02699930600959340

Naghshizadian, R., Rahnemai-Azar, A. A., Kella, K., Weber, M. M., Calin, M. L., Bibi, S., \& Farkas, D. T. (2014). Patient perception of ideal body weight and the effect of body mass index. Journal of Obesity, 2014, 491280. doi:10.1155/2014/491280

Rousseau, A., Knotter, A., Barbe, P., Raich, R. M., \& Chabrol, H. (2005). Étude de validation de la version française du Body Shape Questionnaire. L'Encéphale, 31, 162-173. doi:10.1016/S00137006(05)82383-8

Rousseau, A., Valls, M., \& Chabrol, H. (2010). The Sociocultural Attitudes Towards Appearance Scale 3 (SATAQ-3): Etude de validation de la version française. L'Encéphale, 36, 270-276. doi:10. 1016/j.encep.2008.03.013

Schutz, Y., \& Woringer, V. (2002). Obesity in Switzerland: A critical assessment of prevalence in children and adults. International Journal of Obesity, 26(Suppl. 2), S3-S11. doi:10.1038/sj.ijo. 0802122

Sentilles, R. M., \& Callahan, K. (2012). Beauty over the centuriesFemale. In T. F. Cash (Ed.), Encyclopedia of body image and human appearance (Vol. 1, pp. 43-49). London: Academic Press. 
Stephen, I. D., \& Perera, A. T.-M. (2014). Judging the difference between attractiveness and health: Does exposure to model images influence the judgments made by men and women? PLOS ONE, 9, e86302. doi:10.1371/journal.pone. 0086302

Stice, E. (2002). Sociocultural influences on body image and eating disturbance. In C. G. Fairburn \& K. D. Brownell (Eds.), Eating disorders and obesity: A comprehensive handbook (2nd ed., pp. 103107). New York: Guilford Press.

Stice, E., \& Shaw, H. E. (2002). Role of body dissatisfaction in the onset and maintenance of eating pathology: A synthesis of research findings. Journal of Psychosomatic Research, 53, 985-993. doi:10. 1016/S0022-3999(02)00488-9

Swami, V., Cavelti, S., Taylor, D., \& Tovée, M. J. (2015). The Breast Size Rating Scale: Development and psychometric evaluation. Body Image, 14, 29-38. doi:10.1016/j.bodyim.2015.02.004

Thompson, J. K., van den Berg, P., Roehrig, M., Guarda, A. S., \& Heinberg, L. J. (2004). The Sociocultural Attitudes Towards
Appearance Scale-3 (SATAQ-3): Development and validation. International Journal of Eating Disorders, 35, 293-304. doi:10. 1002/eat. 10257

Tovée, M. J., Emery, J. L., \& Cohen-Tovée, E. M. (2000). The estimation of body mass index and physical attractiveness is dependent on the observer's own body mass index. Proceedings of the Royal Society B, 267, 1987-1997. doi:10.1098/rspb.2000.1240

World Health Organization. (1995). Physical status: The use and interpretation of anthropometry (No. 854). Retrieved from http:// whqlibdoc.who.int/trs/WHO TRS 854.pdf

World Health Organization. (2000). Obesity: Preventing and managing the global epidemic (No. 894). Retrieved from http://whqlibdoc. who.int/trs/WHO_TRS_854.pdf

World Health Organization. (2004). Appropriate body-mass index for Asian populations and its implications for policy and intervention strategies. Lancet, 363, 157-163. doi:10.1016/S0140-6736(03) $15268-3$ 